\title{
LAYER CHARGE AND CHARGE DISTRIBUTION OF SMECTITES: A PARAMETER WHICH CONTROLS IMPORTANT PHYSICAL PROPERTIES OF BENTONITES
}

\author{
Christidis G. E. ${ }^{1}$ \\ ${ }^{1}$ Technical University of Crete, Department of Mineral Resources Engineering, 73100 Chania, \\ Greecechristid@mred.tuc.gr
}

\begin{abstract}
The new method of Christidis and Eberl (2003), which determines the layer charge and charge distribution of dioctahedral smectites in bentonites is presented and is compared to the existing methods for determination of layer charge and charge distribution, namely the structural formula method and the alkylammonium method. The new method is based on the comparison of XRD traces of $K$-saturated, ethyleneglycol solvated smectites with simulated XRD-traces calculated for three-component interlayering. Applications of the method include modeling of the evolution of smectite layers towards illite during diagenesis, and study of the influence of layer charge and charge distribution of smectites on important physical properties of bentonites such as rheological properties (viscosity, gel strength, yield point and thixotropy) and swelling. Smectites with layer charge between -0.425 and -0.47 to 0.48 equivalents phfu affect rheological properties in a different way compared to smectites with higher or lower layer charge than this layer charge interval. Based on these observations a new classification scheme for smectites has been proposed according to their layer charge. In this classification scheme the term smectites with intermediate layer charge has been introduced.
\end{abstract}

Key words: layer charge heterogeneity, bentonites, diagenesis, rheological properties, swelling.

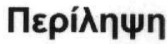

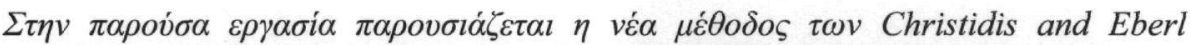

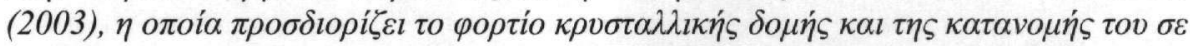

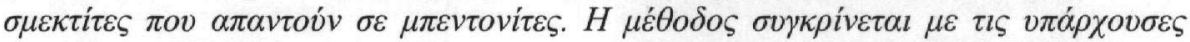

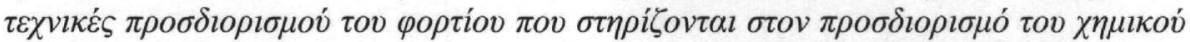

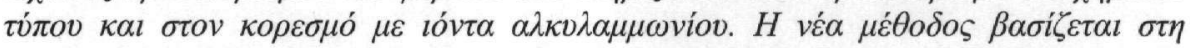

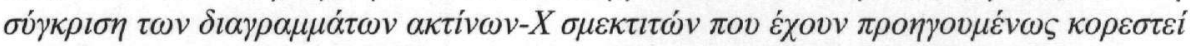

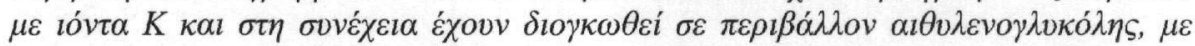

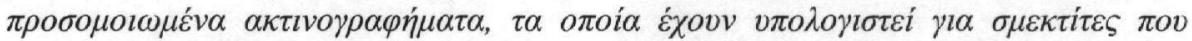

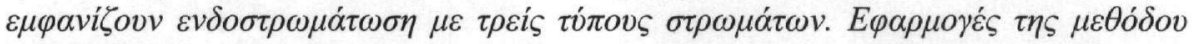

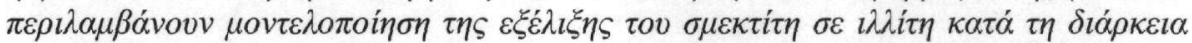

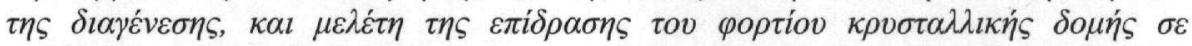

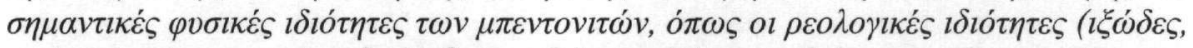

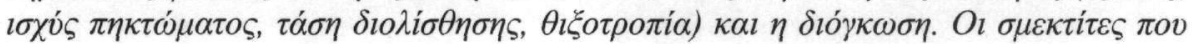




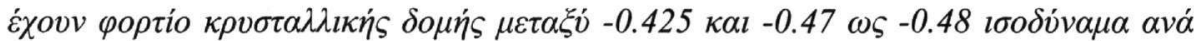

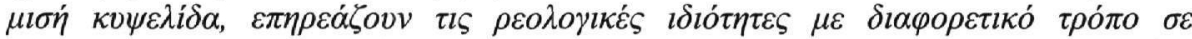

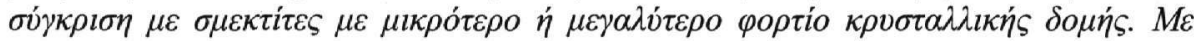

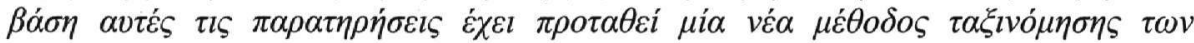

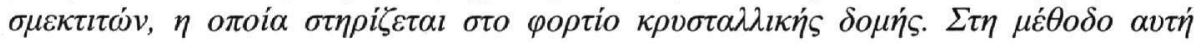

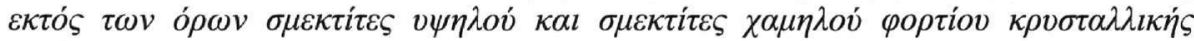

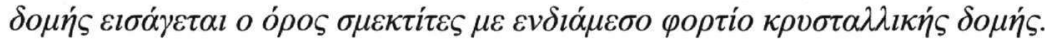

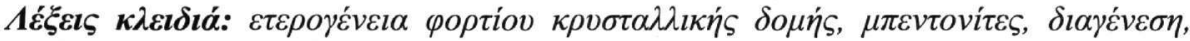

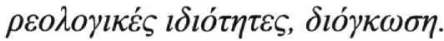

\section{Introduction}

Layer charge is the charge deficit, which stems from ionic substitutions in the structure of minerals. In some minerals the charge is balanced by fixed cations (e.g. $\mathrm{K}, \mathrm{Na}$ and $\mathrm{Ca}$ in micas) whilst in others (e.g. smectite and vermiculite) it is balanced by the so-called exchangeable cations. In the case of smectites characterization of layer charge is of economic and geologic importance, because layer charge strongly affects key smectite properties such as swelling (McEwan and Wilson 1980), cation exchange capacity, and ion exchange selectivity (Maes and Cremers 1977). Smectites often are compositionally and structurally heterogeneous (Stul and Mortier 1974, Lagaly and Weiss 1975, Talibudeen and Goulding 1983, Nadeau et al. 1985, Decarreau et al. 1987, Iwasaki and Watanabe 1988, Lagaly 1994, Christidis and Eberl 2003 among others). This heterogeneity contributes significantly to layer charge heterogeneity, both in terms of charge location (tetrahedral or beidellitic versus octahedral or montmorillonitic charge) and charge magnitude (i.e., individual smectite 2:1 layers may differ in charge, with the total layer charge for a sample being an average of these different charges). Additional factors involved in the structural heterogeneity of smectites include cation ordering in the octahedral sheet, and cis-trans occupancy (Drits et al. 1998, Vantelon et al. 2003, Zviagiva et al. 2004).

Smectite total layer charge and the heterogeneity of this charge from layer to layer can been measured by a variety of methods, including: (1) micro-calorimetry (Talibudeen and Goulding 1983), in which the heat released during determination of an exchange isotherm is related to different types of exchange sites and hence to charge heterogeneity; (2) measurement of the structural formula using chemical or microbeam methods (Weaver and Pollard 1973, Newman and Brown 1987, Christidis 2006), in which the oxide content of a purified smectite sample is measured and then converted into a structural formula; (3) by XRD analysis after saturation with inorganic or organic cations (Tettenhorst and Johns 1966, Cicel and Machajdik 1981, Stul and Mortier 1974, Lagaly 1981, 1994, Olis et al. 1990) and (4) by computer modeling of XRD traces of K-saturated ethylene glycol solvated smectites (Christidis and Eberl 2003). Microcalorimetry does not yield quantitative estimation of the layer charge. Alternative methods for qualitative estimation of smectite layer charge involve $\mathrm{NH}_{4}{ }^{+}$saturation and examination with FTIR (Petit et al. 2006) or examination of ethylene glycol complexes with UV spectroscopy (Bujdak 2006). In this review, which contains also new data, we present some new developments on the determination of smectite layer charge proposed by Christidis and Eberl (2003) we compare them with older existing techniques and present some applications of these developments in clay mineralogy, clay geology and colloidal properties of smectites. Also the influence of these developments on the classification of smectites according to the layer charge is shown.

\section{Existing methods for calculation of layer charge and charge distibution of smectites}

Currently there are two methods used for quantitative determination of layer charge and charge heterogeneity of dioctahedral smectites: The structural formula method and the akylammonium method. 


\subsection{The structural formula method}

The structural formula for expanding $2: 1$ phyllosilicates is calculated from knowledge of the cation composition. Three steps are required: Determination of gram equivalents for each cation according to the following formula

$$
G_{i}=\frac{\mathrm{C}_{\mathrm{izi}}}{\mathrm{AW}_{\mathrm{i}}}
$$

where $\mathrm{G}_{i}=$ gram equivalents for cation i per $\mathrm{g}$ of clay, $\mathrm{z}_{i}=$ valence of the cation and $\mathrm{AW}_{i}=$ atomic weight of the cation.

b) Calculation of estimates of the number of moles of each cation per mole of formula unit.

$$
\mathrm{B}_{\mathrm{i}}=\frac{\mathrm{G}_{\mathrm{i}} \mathrm{F}}{\mathrm{z}_{\mathrm{i}} \sum \mathrm{G}_{\mathrm{i}}}
$$

where $\mathrm{F}$ is the framework of anionic charge (-22.000 moles of charge per mole of formula unit).

c) Finally, cations are assigned 'to tetrahedral, octahedral and interlayer sites according to Pauling's rules

chemical analysis of the clay fraction (usually $<2 \mu \mathrm{m}$ fraction), from electron microprobe analysis and from analytical electron microscopy (AEM). The method can determine the octahedral and tetrahedral charge of smectites but it cannot determine layer charge heterogeneity within the clay fraction. Moreover it can provide information on the chemical heterogeneity of smectites in the microscale (Christidis 2006). Although very often analyses are affected by the presence of impurities, determination of total layer charge with the structural formula method is not affected by contamination from impurities (Laird 1994). In contrast, in some occasions it is difficult to determine accurately the tetrahedral octahedral, permanent and variable octahedral charge by this method.

\subsection{The alkylammonium method}

The method has been developed in the beginning of 60 's. Since then several refinements have been proposed. Alkylammonium clay derivatives are prepared by stoichiometric exchange of interlayer cations of 2:1 phyllosilicates with straight chain alkylammonium ions:

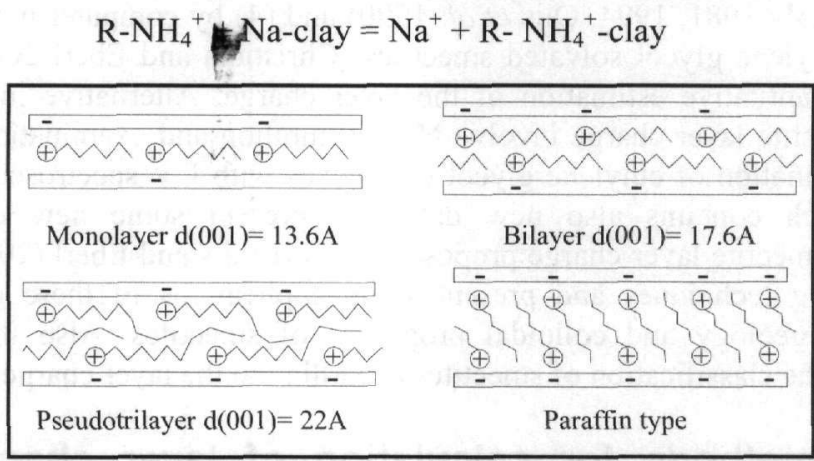

Figure 1 - Confguration of the alkylammonium ions in the interlayer space of smectites

Alkylammonium cations are arranged with monolayer, bilayer, pseudo-trilayer or paraffin-type configurations in the interlayer space according to the carbon chain length $\left(\mathrm{n}_{\mathrm{C}}\right)$ and the layer charge of the clay (Fig. 1). Basal spacings of $13.6 \AA, 17.6 \AA$ and $22 \AA$ indicate monolayer, bilayer 
and pseudotrilayer configurations respectively. In paraffin-type configurations basal spacings vary linearly with $\mathrm{n}_{\mathrm{c}}$. The method uses either the critical carbon chain length associated with monobilayer transitions (most usually applied) or the critical carbon chain length associated with bilayer-pseudotrilayer transitions or the slope of the carbon chain length - basal spacing relationship for samples with paraffin-type interlayer alkylammonium configurations.

Alkylammonium-clay derivatives shift from monolayer to bilayer configuration, when the average planar area for each alkylammonium ion $\left(\mathrm{A}_{c}\right)$ exceeds the average planar area available per charge site $\left(A_{e}\right)$ in the interlayer space. $A_{c}\left(\AA^{2}\right)$ is estimated from:

$$
\mathrm{Ac}_{\mathrm{c}}=5.67\left(n_{c}\right)+14
$$

and $\mathrm{A}_{\mathrm{e}}$ is estimated from:

$$
\mathrm{Ae}=\frac{\mathrm{ab}}{2 \sigma}
$$

where $\mathrm{ab}$ is the planar area per unit cell $\left(46.5 \AA^{2}\right)$ and $\sigma$ is layer charge $\left(\right.$ mole $\left._{\mathrm{c}} \cdot \mathrm{mol}_{\mathrm{fu}}{ }^{-1}\right)$. Then layer charge per half unit cell (phuc) can be calculated from:

$$
\sigma=\frac{23.25}{5.67(n c)+14}
$$

$\mathrm{X}$-ray diffraction patterns of smectites saturated with alkylammonium salts with $\mathrm{n}_{\mathrm{C}}=13,14$ and 15 exhibit intermediate d-spacings between 13.6 (monolayer) and $17.6 \AA$ (bilayer). Such spacings are interpreted as evidence for random interstratification of lower- charge layers with monolayer configurations and higher charge layers with bilayer configurations. By estimating proportions of monolayers and bilayers associated with each sample showing intermediate spacings, the layer charge is subdivided into various classes. Layer charges are $0.285,0.270,0.255$ and 0.243 for the $12-13,13-14,14-15$ and $15-16 \mathrm{n}_{\mathrm{c}}$ density classes respectively.

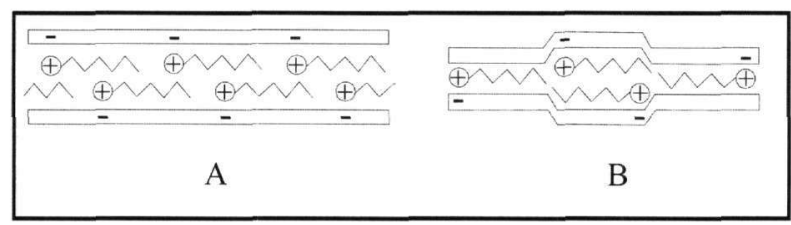

Figure 2 - Configurations of alkylammonium cations in the interlayer space of smectites (modified after Laird 1994)

There are two main caveats in the alkylammonium method (Laird 1994): Since more than $45 \%$ of the interlayer volume of smectites in bilayer configuration of alkylammonium is empty, two types of alkylammonium arrangement can be visualized for $n_{c} \geq 8$ (Fig. 2). In configuration (A) a bilayer formation is favoured, but in configuration (B) the alkylammonium ions and silicate layers are rearranged so as to form islands of bilayers surrounded by monolayers. Hence smectites with homogeneous charge distributions will exhibit gradual mono-bilayer transition. Also alkyl chains of alkylammonium cations located near lateral edges of smectite crystals may protrude out of the interlayer space. Hence the average planar area occupied by an alkylammonium ion on a particle surface is less than predicted by Equation 3 and the layer charge predicted by Equation 5 will underestimate layer charge. This effect is more pronounced in smaller crystals. Indeed it has been demonstrated clearly that the alkylammonium method underestimates the layer charge (Laird et al. 1989, Nikitaki and Christidis unpublished data).

\subsection{The method of Christidis \& Eberl (2003)}

Recently, Christidis and Eberl (2003) presented a novel method for determination of layer charge and charge distribution of smectites. The method is based on the comparison of XRD traces of K- 
saturated, ethylene-glycol solvated smectites with simulated XRD-traces calculated for threecomponent interlayering (fully expandable $17.1 \AA$ layers, partially expandable, $13.5 \AA$ layers and non-expandable $9.98 \AA$ layers). Measurement of layer charge and charge distribution is possible by means of the LayerCharge computer code (Eberl and Christidis 2002). The program finds the calculated pattern which minimizes the sum of square differences between: a) the experimental and calculated peak positions for the first $600 \mathrm{l}$ reflections or b) the whole pattern fit of experimental and calculated intensities normalized to the intensity of the most intense peak. Then it assigns the proportion of $17.1 \AA / 13.5 \AA / 9.98 \AA$ layers (i.e. charge heterogeneity) and calculates the layer charge.

Christidis and Eberl (2003) classified the smectites they studied in 2 groups according to their XRD characteristics. Group 1 smectites have first order reflection between 16 and $17.1 \AA$, and usually well defined higher order basal reflections. They were further subdivided into Group 1a smectites, which are typical low charge smectites, with $001 \mathrm{~d}$-spacing $>16.6 \AA$, and well-defined and rational higher order basal reflections, and Group $1 \mathrm{~b}$ smectites with $001 \mathrm{~d}$-spacing values 16 $16.6 \AA$, irrational higher order basal reflections. In the latter smectites the 002 diffraction maximum can be a shoulder. Group 2 smectites are characterized by $001 \mathrm{~d}$-spacing values $15.5 \AA$ $13.5 \AA$ and a 003 diffraction maximum at 4.65-4.70 $\AA$ which tails off with a shoulder at the low angle side. Usually the 002 diffraction maximum is missing. They are the typical high charge smectites. The main difference between the high and low charge smectites is the proportion of the $17 \AA$ (i.e. low charge) layers. These characteristics have been utilized by Christidis et al. (2006) to propose a classification of smectites according to the layer charge (see below).

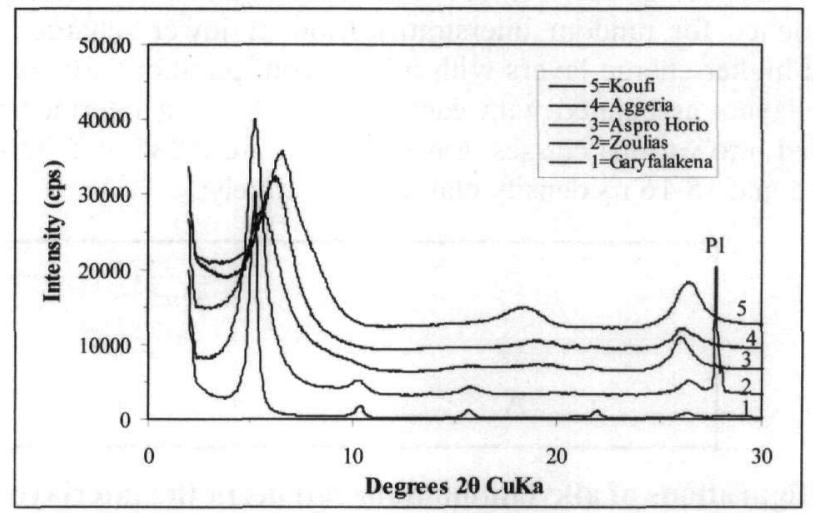

Figure 3 - XRD traces of various Miloan smectites. Traces 1,2 correspond to Group 1a (low charge) smectites, trace 3 corresponds to Group $1 \mathrm{~b}$ smectites and traces 4,5 correspond to Group 2 (high charge) smectites

An example for the variability of smectites from Miloan bentonite deposits according to their layer charge, determined according to the method of Christidis and Eberl (2003) is shown in Figure 3. According to their XRD traces the smectites from the Garyfalakena and Zoulias deposits are low charge smectites $(-0.40$ and -0.42 charge equivalents phfu respectively) and those from the Koufi and Aggeria deposits are high charge smectites ( -0.61 and -0.56 charge equivalents phfu respectively). The smectite from Aspro Horio deposit has layer charge- 0.44 charge equivalents phfu.

\section{Applications of the new method}

\subsection{Diagenesis}

Applications of the new method in clay mineralogy and clay geology have been presented and discussed by Christidis and Eberl (2003). More specifically they showed that the smectite layers 
with intermediate charge are not true vermiculite layers and those smectite layers with high layer charge are not true illite layers. Some novel applications of this method on clay geology not published before concern the evolution of smectite layer charge and the various types of smectites layers with increasing degree of diagenesis. More specifically we applied the method on a data set with R0 mixed layer illite-smectite (IS) from the Silesian basin, Poland used by Srodon et al. (1986). Only samples with smectite contents greater than $50 \%$ were eventually used, because higher abundances of illite layers in mixed-layer IS yielded R1 (i.e. ordered) interstratification (Srodon et al. 1986). For lower smectite (i.e. higher illite) contents we observed collapse of some of the smectite layers after K-saturation and ethylene glycol solvation, presumably due to their high charge. The results are shown in Figure 4.

It is evident that with increasing degree of diagenesis expressed by decreasing of the fraction of expandable (i.e. smectite) layers or by increasing of the amount of fixed $\mathrm{K}$, which is proportional to the percentage of illite layers a) the proportion of $17.1 \AA$ layers (i.e. fully swelling layers) gradually decreases b) the proportion of the $9.98 \AA$ layers (i.e. non swelling layers) increases and c) the proportion of the $13.5 \AA$ layers (i.e. partially swelling layers) follows the same trend as the fully swelling layers. Therefore diagenesis proceeds with formation of layers with gradually increasing layer charge, associated with a gradual disappearance of the swelling character of the 2:1 layer silicates. It is not certain if the increasing proportion of non-swelling layers is associated with formation of beidellitic (i.e. tetrahedrally charged) layers at the expense of montmorillonitic (i.e. octahedrally charged) layers (cf. Beaufort et al. 2001), or merely by gradual decrease of the octahedral charge only via release of $\mathrm{Mg}$. This is because we have shown that the proportion of tetrahedrally charged layers affects the determination of layer charge (Christidis 2004).

Previous work on diagenetic changes in North Sea sediments based on modelling of XRD traces has reported the formation of vermiculitic layers during the smectite-to-illite formation (Drits et al. 1997). In that work the term vermiculite layers was used to describe the formation of partially swelling layers in the mixed layer phase. However Christidis and Eberl (2003) have shown that the vermiculite layers are different from high charge smectite layers and they can be distinguished. In this sense, using the finding of this work it is proposed that the conversion of smectite-to-illite may not involve formation of vermiculite layers but of high charge smectite layers. As noted before, these high charge layers may well belong to tetrahedrally charged smectites.
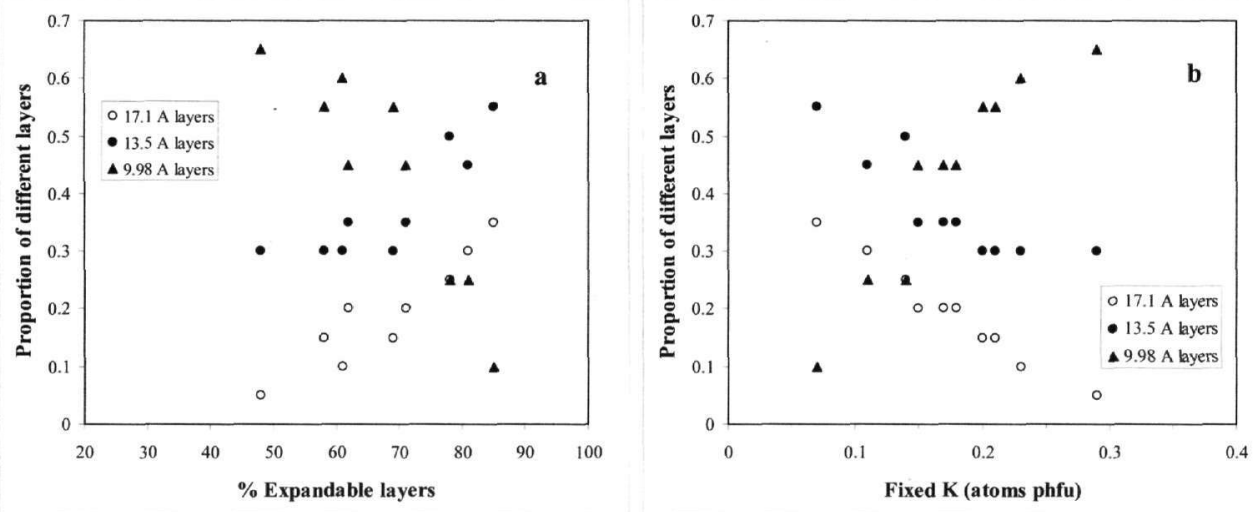

Figure 4 - Evolution of the various types of smectite layers in R0 mixed layer IS from the Silesian Basin, Poland, as a function (a) of the proportion of expandable layers and (b) of the number of fixed K-atoms. Data for \% of expandable layers and fixed K-atoms in IS are from Srodon et al. (1986) 


\subsection{Rheological and swelling properties}

Rheological properties of bentonites such as viscosity, gel strength and yield point are important for the drilling industry. Similarly swelling is important for civil engineering applications, foundry industry and environmental applications. The industry uses Na-bentonites, which have considerably higher swelling capacities compared to their Ca-Mg-counterparts. This is why the most common upgrading process used in $\mathrm{Ca}$-bentonites is Na-activation, which involves replacement of $\mathrm{Ca}$ exchangeable cations by $\mathrm{Na}$.

Recently Christidis et al. (2006) showed that viscosity, gel strength and thixotropic behaviour of bentonites are related to the layer charge of the smectites present. A similar relationship was observed for swelling. Also it was found that both rheological and swelling properties depend on the fraction of the fully swelling layers and that swelling is reversibly related to the fraction of the non swelling layers. Although such dependence is expected, due to the influence of the layer charge on the electroviscous effect and the thickness of quasicrystals of smectites it had not been demonstrated in the past.

According to Christidis et al. (2006) the rheological properties of bentonites do not vary in the same manner over the layer charge range -0.38 to -0.60 equivalents phfu in smectites. More specifically, smectites with layer charge lower than -0.425 (classified as low charge smectites-see next section) develop viscous dispersions with high gel strength, often thixotropic, but no specific relationship was observed between the rheological properties and layer charge. Similarly, smectites with layer charge greater than -0.49 to -0.50 equivalents phfu (classified as high charge smectites-see next section) in general yield dispersions with low viscosity and low gel strength, but again no specific relationship was observed between the rheological properties and layer charge. In contrast in smectites with layer charge between -0.425 and -0.47 to -0.48 equivalents phfu a clear negative trend was observed between layer charge and rheological properties and swelling. The aforementioned different trends will assist to the understanding of the so far unexplained variability of the bentonite deposits in terms of rheological and swelling properties. This topic is currently under investigation.

\section{Classification of smectites according to layer charge}

The proposed method has been used to classify smectites according to the layer charge because so far although the terms high charge and low charge smectites are used extensively in the literature, there is no actual definition about the exact meaning of these terms (cf. Christidis et al. 2006). The classification scheme uses the characteristics of XRD-traces of K-saturated ethylene glycol solvated smectites and is corroborated by the colloidal properties of the various types of smectites. More specifically low charge smectites with layer charge less than -0.425 phfu yield distinct XRD traces as do smectites with layer charge greater than -0.470 phfu (Christidis and Eberl 2003). These layer charge boundaries separate another type of smectite with distinct XRD characteristics, in which important rheological properties are related to the variation of layer charge (Christidis et al. 2006). Thus, between the typical low charge smectites and those smectites with layer charge greater than $-0.47--0.49 \mathrm{phfu}$, there is a group of smectites in which rheological properties vary proportionally to the layer charge.

The classification scheme proposes more functional definitions for the high and low charge dioctahedral smectites (Fig. 5). Thus, the low charge smectites must have, in general, layer charge lower than -0.425 equivalents phfu for montmorillonite $(-0.365$ equivalents phfu for beidelllite and nontronite because tetrahedrally charged smectites yield XRD traces like montmorillonite with fewer low charge layers), and XRD traces with first order reflections (for K-saturated, glycolated samples) that range from 16.6 to $17.1 \AA$, and well defined rational higher order basal reflections (traces 1,2 in Fig. 1). Such smectites consist predominately of low charge (17 $\AA$ ), fully swelling layers, develop suspensions with high viscosity and have high swelling volumes. Trioctahedral hectorite also has similar layer charge and XRD characteristics and develops suspensions with 
high viscosity. High charge dioctahedral smectites must have layer charge of at least -0.47 equivalents phfu ( -0.415 equivalents phfu for beidelllite and nontronite) and XRD traces characterized by a first order maximum at $14 \AA$ and a 003 diffraction maximum at $4.6-4.7 \AA$ (traces 4,5 in Fig. 1). Such smectites contain a significant proportion of high charge $(9.98 \AA)$, nonswelling layers, they develop suspensions with low viscosity and have low swelling volumes (Christidis et al. 2006). Finally those smectites with layer charge between -0.425 and -0.47 equivalents phfu ( -0.365 and -0.415 equivalents phfu for beidellite and nontronite), which have XRD traces characterized by first order reflections from 16.6 to $16.0 \AA$ and irrational higher order basal reflections, will be defined as smectites with intermediate layer charge (trace 3 in Fig. 1). Note that the smectites with intermediate layer charge typically are equivalent to group $1 \mathrm{~b}$ smectites of Christidis and Eberl (2003). As noted before the colloidal properties of these smectites vary according to the layer charge and the proportion of the low charge layers.

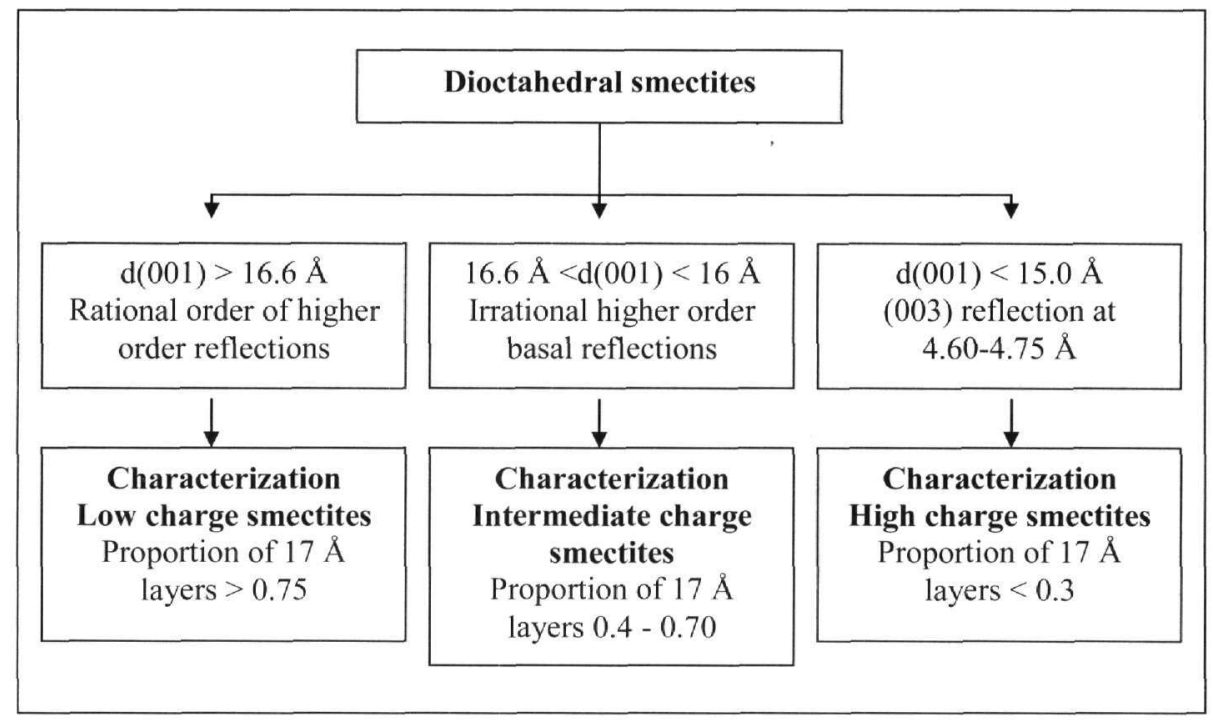

Figure 5 - Proposed classification scheme of smectites according to their layer charge, based on their XRD characteristics

\section{Conclusions}

Layer charge is an important property of smectites, which affects most physical properties of this mineral. We have proposed a method for determination of layer charge of smectites based on the XRD characteristics of K-saturated, ethylene glycol solvated smectites. Application of this method has led to a new functional classification scheme for smectites according to their layer charge. The proposed classification scheme is corroborated by the rheological and swelling properties of smectites. According to this scheme low charge smectites have layer charge smaller than -0.425 phfu and high charge smectites have layer charge greater than -0.470 phfu. These layer charge boundaries separate the smectites with intermediate layer charge. The new method of layer charge characterization of smectites has several applications in clay mineralogy, clay geology and physical properties of bentonites. An important novel application in clay geology is the distribution of layer charge in bentonite deposits, which will shed light to the mechanism of formation of bentonites. Such work is currently in progress.

\section{Acknowledgements}

Financial support from the Fulbright Institution and the Greek Secretariat of Research and Development is greatfully acknowledged. 


\section{References}

Beaufort, D., Berger, G., Lacharpagne, J.C., and Meunier A., 2001. An experimental alteration of montmorilonite to a di + trioctahedral smectite assemblage at 100 and $200^{\circ} \mathrm{C}$, Clay Minerals, 36, 211-225.

Bujdak, J., 2006. Effect of the layer charge of clay minerals on optical properties of organic dyes, A review. Applied Clay Science, 34, 58-73.

Christidis, G.E., 2004. A new method for the determination of layer charge and charge distribution of smectites, International workshop on current knowledge on the layer charge of clay minerals, program and abstracts, Smolenice, Slovakia.

Christidis, G.E., 2006. Genesis and compositional heterogeneity of smectites. Part III: Alteration of basic pyroclastic rocks-A case study from the Troodos ophiolite Complex, Cyprus, American Mineralogist, 91, 685-701.

Christidis, G.E., and Eberl, D.D., 2003. Determination of layer charge characteristics of smectites, Clays and Clay Minerals, 51, 644-655.

Christidis, G.E., Blum, A.E., and Eberl, D.D., 2006. Influence of layer charge and charge distribution of smectites on the flow behaviour and swelling of bentonites, Applied Clay Science, 34, 125-138.

Cicel, V., and Machajdik, D., 1981. Potassium- and amnonium-treated montmorillonites. I. Interstratified structures with ethylene glycol and water, Clays and Clay Minerals, 29, 4046.

Cuadros, J., Sainz-Diaz, C.I., Ramirez, R., and Hernandez-Laguna, A., 1999, Analysis of Fe segregation in the octahedral sheet of bentonitic illite-smactite by means of FTIR, ${ }^{27} \mathrm{Al}$ MAS NMR and reverse Monte Carlo simulations, American Journal of Science, 299, 289308.

Decarreau, A., Colin, F., Herbillon, A., Manceau, A., Nahon, D., Paquet, H., Trauth-Badeaud, D., and Trescases, J.J., 1987. Domain segregation in Ni-Fe-Mg-smectites, Clays and Clay Minerals, 35, 1-10.

Drits, V.A., Sakharov, B.A., Lindgreen, H., and Salyn A., 1997. Sequential structure transformation of illite-smectite-vermiculite during diagenesis of Upper Jurassic shales from the North Sea and Denmark, Clay Minerals, 32, 351-371.

Drits, V.A., Lindgreen, H., Salyn, A.L., Ylagan, R., and McCarty, D.K., 1998. Semi quantitative detrmination of trans-vacant and cis-vacant 2:1 layers in illites and illite-smectites by thermal analysis and X-ray diffraction, American Mineralogist, 83, 1188-1198.

Eberl, D.D., and Christidis, G., 2002. LayerCharge: A computer program for calculation of layer charge and charge distribution of smectites, USGS, Boulder, Colorado.

Iwazaki, T., and Watanabe, T., 1988. Distribution of $\mathrm{Ca}$ and $\mathrm{Na}$ ions in dioctahedral smectites and interstratified dioctahedral mica/smectites, Clays and Clay Minerals, 36, 73-82.

Lagaly, G., 1981. Characterization of clays by organic compounds, Clay Minerals, 16, 1-21.

Lagaly, G., 1994. Layer charge determination by alkylammonium ions. In A.R.Mermut (ed.), Layer charge characteristics of 2:1 silicate clay minerals, 2-46pp., CMS Workshop lectures, 6, The Clay Minerals Society, Boulder Colorado.

Lagaly, G., and Weiss A., 1975. The layer charge of smectitic layer silicates, Proceedings International Clay Conference Mexico, 157-172. 
Laird, D.A., 1994. Evaluation of the structural formula and alkylammonium methods of determining layer charge. In A.R.Mermut (ed.), Layer charge characteristics of 2:1 silicate clay minerals, 80-103pp., CMS Workshop lectures, 6, The Clay Minerals Society, Boulder Colorado.

Laird, D.A., Scott, A.D., and Fenton, T.E., 1989. Evaluation of the alkylammonium method of determining layer charge, Clays and Clay Minerals, 37, 41-46.

MacEwan, D.A.C., and Wilson, M.J., 1984. Interlayer and intercalation complexes of clay minerals. In G.W. Brindley and G. Brown (eds), Crystal structures of clay minerals and their X-ray identification, 197-248pp., Mineralogical Society, London.

Maes, A., and Cremers, A., 1977. Charge density effects in ion exchange. Part 1. Heterovalent exchange equilibria, Faraday Transactions of the Royal Chemical Society, 73, 1807-1814.

Nadaeu, P.H, Farmer, V.C., McHardy, W.J., and Bain, D.C., 1985. Compositional variations of the Unterrupsroth beidellite, American Mineralogist, 70, 1004-1010.

Newman, A.C.D., and Brown, G., 1987. The chemical constitution of clays. In A.C.D Newman, (ed.), Chemistry of Clays and Clay Minerals 1-128, Mineralogical Society, London.

Olis, A.C., Malla, P.B., and Douglas, L.A., 1990. The rapid estimation of the layer charges of 2:1 expanding clays from a single alkylammonium ion expansion, Clay Minerals, 25, 39-50.

Petit, S., Righi, D., and Madejova, J., 2006. Infrared spectroscopy of $\mathrm{NH}_{4}{ }^{+}$-bearing and saturated clay minerals: A review of the study of layer charge, Applied Clay Science, 34, 22-30.

Srodon, J., Morgan, D.J., Eslinger, E.V., Eberl., D.D., and Karlinger, M.R., 1986. Chemistry of illite/smectite and end-member illite, Clays and Clay Minerals, 34, 368-378.

Stul, M.S., and Mortier, W.J., 1974. The heterogeneity of the charge density in montmorillonites, Clays and Clay Minerals, 22, 391-396.

Talibudeen, O., and Goulding, K.W.T., 1983. Charge heterogeneity in smectites, Clays and Clay Minerals, 31, 37-42.

Tettenhorst, R., and Johns, W.D., 1966. Interstratification in montmorillonite, Clays and Clay Minerals, 15, 85-93.

Vantelon, D., Montarges-Pelletier, E., Michot, L.J., Briois, V., Pelletier, M., and Thomas, F., 2003. Iron distribution in the octahedral sheet of dioctahedral smectites. An Fe K-edge Xray absorption spectroscopy study, Physics and Chemistry of Minerals, 30, 44-53.

Weaver, C.E., and Pollard, L.D., 1973. The chemistry of clay minerals, 55-77pp., Elsevier, Amsterdam.

Zviagina, B.B., McCarty, D.K., Srodon, J., and Drits, V.A., 2004. Interpretation of infrared spectra of dioctahedral smectites in the region of $\mathrm{OH}$-stretching vibrations, Clays and Clay Minerals, 52, 399-410. 